\title{
EVALUACIÓN DE LAS ESTADÍSTICAS DE COLAPSO DE PUENTES EN COLOMBIA POR CARGAS EXPLOSIVAS
}

\section{STATISTICAL ASSESSMENT OF BRIDGE COLLAPSE IN COLOMBIA BY BLAST LOADING}

\author{
Julián Carrilloº, Laura Arciniegas Londoño², Jesika Alejandra Molina Figueroa ${ }^{3}$
}

Fecha de recepción: 1 de agosto de 2014

Fecha de aprobación: 6 de octubre de 2014

Referencia: J. Carrillo, L. Arciniegas Londoño, J.A. Molina Figueroa. (2014). Evaluación de las estadísticas de colapso de puentes en Colombia por cargas explosivas. Ciencia e Ingeniería Neogranadina, 24 (2), pp. 157 - 175

\section{RESUMEN}

En el artículo se presentan y discuten los datos registrados del colapso de puentes en Colombia por cargas explosivas originadas por atentados terroristas entre 1994 y 2014 . El estudio incluye un análisis estadístico en términos de la ubicación, las características, el tipo y material con el cual estaban construidos los puentes. Este análisis demostró que la mayoría de los puentes colapsados se encontraban en lugares que actualmente son clasificados como «zonas rojas» del país. El estudio también incluye el análisis de los impactos económicos, sociales y políticos ocasionados por el colapso del puente ubicado sobre el río Riecito en el Caquetá. Este puente fue rehabilitado por medio de puentes móviles tipo Acrow pertenecientes al Ejército Nacional de Colombia e instalado y supervisado por el Instituto Nacional de Vías, INVIAS.

Palabras Clave: puentes, colapso, carga explosiva, economía

\section{ABSTRACT}

The recorded data of bridge collapse in Colombia by blast loading caused by terrorist attacks, from 1994 to 2014 is presented and discussed in this paper. The study includes a statistical analysis of the location, characteristics, type, and construction materials of the bridges. This analysis demonstrates that most of the collapsed bridges were located at places that are currently classified as "red zones" in the country. The study also includes the analysis of the economic, social

1. Ingeniero Civil, Ph.D. Profesor e Investigador, Programa de Ingeniería Civil. Universidad Militar Nueva Granada, Bogotá D.C., Colombia, wjcarrillo@ gmail.com

2. Profesional en Ciencias Militares, Auxiliar de Investigación, Programa de Ingeniería Civil. Universidad Militar Nueva Granada, UMNG, Bogotá D.C., Colombia, laura_arci@hotmail.com

3. Profesional en Ciencias Militares, Auxiliar de Investigación, Programa de Ingeniería Civil. Universidad Militar Nueva Granada, UMNG, Bogotá D.C., Colombia, jealmofi@hotmail.com 
and political impact triggered by the collapse of the bridge located on the Riecito River in Caquetá. This bridge was rehabilitated using Acrow-type bridges that are employed by the National Army of Colombia and are installed and supervised by the National Institute of Roads, INVIAS.

Keywords: bridges, collapse, blast loading, economy

\section{INTRODUCCIÓN}

De acuerdo con Leal [1], desde la década de los 80, Colombia ha experimentado atentados con explosivos que han dejado decenas de muertos y cientos de heridos; es decir, han sido más de 30 años cohibidos y afectados por el conflicto interno. Además del terrorismo, las acciones de los grupos al margen de la ley respaldan una estrategia conocida como «sabotaje», la cual se desarrolla con la activación de explosivos, carros bomba y campos minados, así como la voladura de puentes y ataques a la infraestructura petrolera y energética del país. Entre las estructuras más afectadas, los puentes han sido objetivo de gran interés y blanco estratégico de los grupos terroristas, pues su colapso y rehabilitación no sólo genera un desorden social, sino un desgaste a las propias tropas y a los recursos dirigidos a la prevención y atención de desastres del gobierno nacional.

Según Muñoz [2], las estadísticas de años anteriores a 1997 demuestran que la afectación de los puentes por atentados terroristas representa el $31 \%$ de los colapsos generales en Colombia. Este es un porcentaje considerablemente alto y que no ha disminuido, pues en el periodo que se evalúa en este artículo (1994 - 2014), los puentes siguen siendo una estructura estratégica de impacto, cuyo colapso está dirigido no sólo a la población sino también a la manera operacional del Ejército de Colombia. De acuerdo con el Instituto de Cooperación para la Seguridad Hemisférica [3], en este tipo de atentados se utiliza casi la misma doctrina de explosivos y en su acto delictivo se deteriora significativamente la infraestructura vial y energética del país, lo cual genera impactos sociales, económicos y políticos en la región afectada. Estos impactos deben tenerse en cuenta para establecer una posible solución, que en la mayoría de los casos abarca el remplazo del puente por uno temporal y con características suficientes para el paso de un lugar a otro, pero sin incluir las características que tenía el puente antes de ser derribado. Por ejemplo, el Puente Riecito fue derribado y su rehabilitación no contempló las capacidades (carga y número de carriles) que la estructura disponía antes de su colapso.

Para evaluar los datos registrados del colapso de puentes en Colombia en el periodo de 1994 a 2014, inicialmente se destacan los puntos clave que determinan al puente como puesto estratégico terrorista. Luego se analizan las estadísticas generales recopiladas desde 1997 hasta 2014, resaltando no sólo los lugares más frecuentes de este tipo de atentados terroristas, las características, el tipo y material con el cual estaban construidos los puentes, sino también las características de las cargas explosivas, la efectividad y la ubicación estratégica que se emplea para el colapso del puente, y los impactos sociales y económicos implícitos en la falla. Por último se incluyen los resultados de un reconocimiento detallado al puente Riecito, donde se discute la forma delictiva y la evaluación de los parámetros de estudio. 
El estudio tiene una extensión territorial que abarca principalmente las «zonas rojas» en Colombia, concentradas en los sectores de Cundinamarca, Meta, Antioquia, Caquetá, Tolima, Cesar, Cauca, Nariño, Huila, Boyacá y Guaviare. Los datos presentados sobre el colapso de puentes y su método delictivo para la destrucción del mismo fueron recolectados de información proporcionada por INVIAS, la Jefatura de Ingenieros Militares y de las noticias recurrentes sobre atentados terroristas por explosivos.

Teniendo en cuenta que la información operacional de las tropas colombianas no puede ser revelada ni siquiera a personal militar que se desempeñe fuera de los cargos de jefes de operación, el estudio no incluye datos específicos de la inteligencia militar colombiana, y sólo se clarifican los aspectos que por doctrina son reconocidos y los daños que sin duda alguna no pueden ocultarse a la crítica y necesidad de la población civil.

\section{CAUSAS PRINCIPALES DE ATENTADOS CONTRA PUENTES}

Para Sánchez Cuenca [4], el curso de acción tomado por los grupos terroristas de Colombia se centra básicamente en crear terror e impacto psicológico para desmotivar a la población civil. Este tipo de operación terrorista se conoce como sabotaje, que consiste en realizar un altercado o modificaciónenuna operaciónajena, generando conflicto pero con un «beneficio» a los grupos terroristas. Estas acciones tienen intención de daño y comprometen no sólo al personal de soldados que enfrentan sus actos en primera línea, sino a aquel papel importante que juegan los puentes en la conectividad de las regiones, que incluye acortar distancias, disminuir tiempos de desplazamiento y reducir costos operativos en el transporte de personal y alimentos. Estas características de funcionamiento resaltan la importancia de los puentes y los hacen llamativos para cometer un acto terrorista. A continuación se discuten las principales razones por las cuales los puentes son los blancos principales para este tipo de atentados.

\subsection{AFECTAR LA ECONOMÍA DEL PAís}

Los recursos generados para la prevención y atenciónadesastresporpartedelaOrganización Colombia Humanitaria son generalmente sumas de dinero significativamente altas. Estos recursos se asignan con la intención de reparar daños ocasionados por cambios climáticos y comportamiento no esperado de la naturaleza. Sin embargo, como se muestra en la Tabla 1, en muchas ocasiones estos recursos tienen que ser redireccionados a departamentos como Cundinamarca, Meta, Antioquia, Caquetá, Tolima, Cesar, Cauca, Nariño, Huila, Boyacá y Guaviare, que son los departamentos que más

Tabla 1. Resumen del impacto del terrorismo en Colombia.

\begin{tabular}{|c|c|c|}
\hline $\begin{array}{c}\text { Promedio } \\
\text { anual de } \\
\text { atentados }\end{array}$ & $\begin{array}{c}\text { Valor promedio } \\
\text { del daño en } \\
\text { millones de } \\
\text { USD }\end{array}$ & $\begin{array}{c}\text { Departamentos más afectados por acciones } \\
\text { terroristas }\end{array}$ \\
\hline 862 & 3.1 & $\begin{array}{c}\text { Antioquia, Arauca, Cundinamarca, Norte de } \\
\text { Santander, Valle del Cauca }\end{array}$ \\
\hline
\end{tabular}

Fuente. Departamento Nacional de Planeación, 2012 [9]. 
sufren por atentados terroristas con daños en la infraestructura vial y energética del país. Por ejemplo, en la Figura 1 se muestran los costos generados por atentados terroristas contra puentes desde 1999 hasta 2002. Este nuevo uso no esperado genera que los dineros del Estado que están inicialmente previstos para inversión social en las regiones no sean suficientes y crean daños colaterales tales como el desplazamiento forzoso y la desvalorización de la zona agricultora de las regiones afectadas.

\subsection{CREAR CONCENTRACIÓN DEL GOBIERNO Y DEL PAÍS PARA DESPROTEGER OTRAS ZONAS}

Cuando ocurre un daño en un puente por atentado terrorista, los altos mandos políticos y militares centran su atención en el análisis y causas del daño, incluyendo el afán de reparación para rehabilitar el bien civil atentado. Según Domínguez [5], en el año 2002, a menos de un mes de iniciar un proceso de negociación de paz con las Fuerzas Armadas Revolucionarias de Colombia, FARC, se produjeron cuatro atentados terroristas en Valle del Cauca, Cauca y la Guajira, distanciados cada uno por un lapso de tiempo no mayor a 20 minutos.

\subsection{AFECTAR A LAS POBLACIONES A SU ALREDEDOR}

Para Durán [6], la conexión de las poblaciones con vías principales y con zonas de importancia para la región es ciertamente denigrada cuando un artefacto explosivo interfiere en la tranquilidad de la sociedad, afectando intencionalmente a toda la población. Una consecuencia de mucha atención es la parte económica, pues el movimiento de las personas a sus lugares de trabajo, estudio y vivienda ya no se encuentra a su alcance y, por tanto, se consigue generar molestia, inseguridad y desconfianza de la población con respecto a la acción reparadora del sector social del Estado.

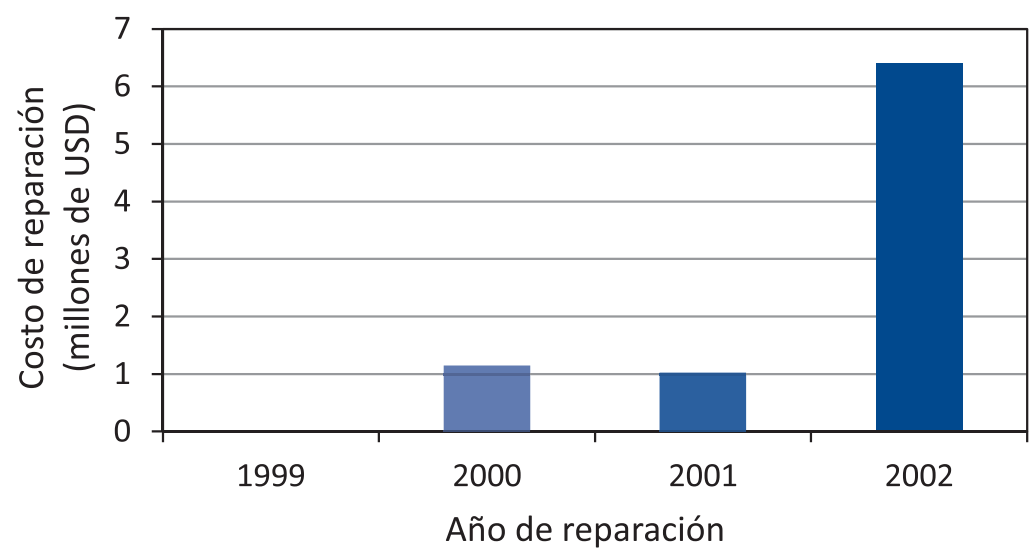

Figura 1. Costos generados por atentados terroristas contra puentes entre 1999 y 2002 en Colombia.

Fuente: INVIAS, 2003 [11]. 


\subsection{CAUSAR DAÑOS DE AVALANCHAS O INUNDACIÓN}

Según Castillo [7], estos factores de inundación o avalanchas dependen del peso y la geometría del puente con relación a la fuerza del caudal del río. Es decir, si el peso del puente es mayor a la fuerza del caudal, la quietud del puente sobre la zona afectada genera un incremento en el nivel del fluido, logrando inundaciones en los sectores aledaños al cauce. Por otro lado, si el peso del puente es menor que la fuerza del caudal, el desplazamiento de los escombros del puente por el cauce del río puede generar derrumbes y avalanchas significativas de tierra, al punto de afectar las viviendas cercanas a la zona de movimiento del río.

\section{ANÁLISIS ESTADÍSTICO DE PUENTES COLAPSADOS POR ACTOS TERRORISTAS EN EL PERIODO DE 1994-2014}

De acuerdo con la campaña del Centro Nacional de Memoria Histórica [8] conocida como «iBasta ya!», el daño a bienes civiles en Colombia se encuentra registrado desde el año de 1988 hasta el 2012. En cifras conocidas se registran 5139 atentados terroristas con artefactos explosivos, de los cuales los puentes son protagonistas no sólo en su acción de colapso sino también como sitios elegidos para quemas de vehículos con el fin de obstruir el paso del puente. De acuerdo con Muñoz [2], en años anteriores a 1997, los puentes colapsados por atentados terroristas registran un 31\% de protagonismo en Colombia. Sin embargo actualmente (2014), la suma de puentes colapsados por explosivos se manifiesta en función del periodo y la época del año, tal como se muestra en la Figura 2, donde se demuestra la gran diferencia numérica dependiendo del año y la época en la cual transcurre el país.

En el año 2002, cuando el expresidente Andrés Pastrana finalizó la zona de distención, los atentados terroristas en Colombia se convirtieron en una ola común y repetitiva. Después del 21 de febrero del año 2002 ocurrieron cifras descomunales. Al comparar estas cifras con periodos anteriores y posteriores se observó que la cantidad de puentes colapsados, según el Departamento

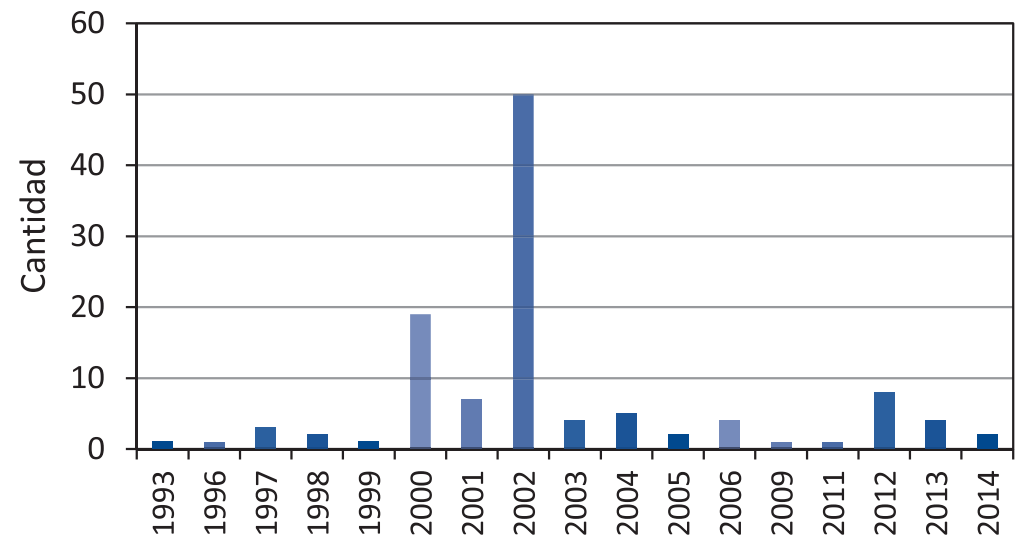

Año

Figura 2. Estadísticas de puentes colapsados por atentados terroristas entre 1993 y 2014. 
Nacional de Planeación [9], fue de 50. Esta cifra fue la más alta y alarmante para institutos como INVIAS, quienes debían recurrir a la rehabilitación en ambientes de guerra y tiempos de alerta roja. En las Tablas 2 y 3 se describen los puentes atentados y colapsados desde 1994 hasta 2014, registrados y no registrados en el Sistema de Administración de Puentes de Colombia, SIPUCOL, respectivamente.

En la Tabla 2, de puentes registrados en SIPUCOL, se indica el nombre del puente, la carretera donde se encuentra ubicado, el departamento, las luces del puente, el ancho del tablero, el material de la superestructura, la estructuración longitudinal y los años de construcción, colapso y rehabilitación. En cuanto al material de la superestructura, los puentes se han clasificado como en concreto $(C)$, acero $(A)$, acero-concreto $(A-C)$, concreto reforzado (CR), concreto presforzado (CP) y mampostería (M). En cuanto a la estructuración longitudinal, los puentes se han clasificado como simplemente apoyado (SA), simplemente apoyado con sección constante (SASC), simplemente apoyado con sección variable (SASV), viga continua con sección constante (VCSC), viga Gerber con sección constante (VGSC), pórtico con sección constante (PSC), y atirantado (A). Según Vallecilla [10], las vigas Gerber se caracterizan por tener articulaciones en determinados sitios a lo largo de la luz, y racionalmente, las articulaciones deben colocarse donde el momento flector de la misma es igual a cero (0). En la Tabla 3, de puentes no registrados en SIPUCOL, sólo se indica el nombre del puente, la carretera donde se encuentra ubicado, el departamento y el año en el cual fue colapsado.

De los 115 puentes atentados y colapsados desde 1994 hasta 2014, en la Tabla 2 se muestran 50 puentes que están registrados en la memoria del SIPUCOL de INVIAS. Los demás puentes se indican en la Tabla 3 y corresponden a los puentes reconocidos por los boletines mensuales generados por el Ministerio de Defensa Nacional. Se concluye que los puentes que no se encuentran registrados en la memoria del SIPUCOL de INVIAS son aquellos que comunican veredas o pequeños municipios en sectores lejanos del centro de los departamentos.

De acuerdo con la cantidad de puentes colapsados por atentados terroristas en el periodo de 1994 hasta 2014, los lugares con mayor frecuencia comprenden los departamentos de Cundinamarca, Meta, Antioquia, Caquetá, Tolima, Cesar, Cauca, Nariño, Huila, Boyacá y Guaviare. Según INVIAS [11], para poder atender emergencias de atentados terroristas, la subdirección de conservación tuvo que direccionar 3,5 millones de USD para la reparación de los puentes que en el año 2002 fueron blanco terrorista, dejando de lado las tareas de reparación y reconstrucción de los puentes de estas zonas.

Según Arango [12], las pérdidas económicas entre los años de 1996 y 2003 varían entre 0,2 y 5,5 millones de USD. Esta inversión se considera como pérdida interna económica, pues se deben reponer los dineros consumidos por la inesperada rehabilitación. En la situación de desarrollo de la nación colombiana, estas grandes sumas de dinero hacen que exista una considerable variación de los recursos generados para los servicios y el bienestar social del país.

De acuerdo con las características de los puentes colapsados por atentados terroristas, en la Tabla 2 y en la Figura 3 se muestra que la luz total de los puentes registrados superaba los $9 \mathrm{~m}$ de largo. Adicionalmente, es común encontrar que la mayoría de estos puentes 
Tabla 2. Puentes atentados y colapsados desde 1994 hasta el 2014 registrados en SIPUCOL.

\begin{tabular}{|c|c|c|c|c|c|c|c|c|c|c|c|c|c|}
\hline \multirow{2}{*}{ No } & \multirow{2}{*}{$\begin{array}{c}\text { Nombre } \\
\text { puente }\end{array}$} & \multirow{2}{*}{ Carretera } & \multirow{2}{*}{ Departam. } & \multicolumn{4}{|c|}{ Luces, m } & \multirow{2}{*}{$\begin{array}{c}\text { Ancho } \\
\text { tablero, } \\
\mathrm{m}\end{array}$} & \multirow{2}{*}{$\begin{array}{l}\text { Mat. } \\
\text { constr. }\end{array}$} & \multirow{2}{*}{$\begin{array}{l}\text { Estruct. } \\
\text { long it. }\end{array}$} & \multicolumn{3}{|c|}{ Año } \\
\hline & & & & $\mathrm{N}$ & Mín. & Máx. & Total & & & & Const. & Colap. & Reh. \\
\hline 1 & Morichito & $\begin{array}{c}\text { Puerto Rico - Yé } \\
\text { de Granada }\end{array}$ & Meta & 1 & - & - & 33.5 & 9.0 & $A C$ & SA & & 1993 & \\
\hline 2 & Villartega & $\begin{array}{c}\text { Chigorodo - } \\
\text { Dabeiba }\end{array}$ & Antioquia & 2 & 25.1 & 48.9 & 74.0 & 9.6 & $\mathrm{CP}$ & SASC & & 1997 & 1997 \\
\hline 3 & $\begin{array}{c}\text { La } \\
\text { Congunta }\end{array}$ & $\begin{array}{c}\text { Sogamoso - Agua } \\
\text { Azul }\end{array}$ & Casanare & 1 & - & - & 24.5 & 3.2 & A & SASC & 1958 & 1997 & \\
\hline 4 & La Granja & $\begin{array}{l}\text { Santuario - Caño } \\
\text { Alegre }\end{array}$ & Antioquia & 1 & - & - & 30.4 & 9 & $\mathrm{CR}$. & VCSC & 1995 & 1997 & \\
\hline 5 & El Viao & $\begin{array}{l}\text { Santuario - Caño } \\
\text { Alegre }\end{array}$ & Antioquia & 3 & 10.3 & 30.8 & 51.7 & 10.5 & $\mathrm{CP}$ & SASC & & 1998 & 1999 \\
\hline 6 & Tasido & $\begin{array}{c}\text { Chigorodó - } \\
\text { Dabei ba }\end{array}$ & Antioquia & 1 & - & - & 30.3 & 11.5 & $\mathrm{CP}$ & SASC & & 1998 & \\
\hline 7 & Nechi & $\begin{array}{l}\text { Medellí n-los } \\
\text { Ilanos }\end{array}$ & Antioquia & 1 & - & - & 50.8 & 10.2 & $\mathrm{CP}$ & PSC & 1956 & 1999 & 2001 \\
\hline 8 & $\begin{array}{c}\mathrm{El} \\
\text { Caminito } \\
\end{array}$ & $\begin{array}{l}\text { Santuario - Caño } \\
\text { Alegre }\end{array}$ & Antioquia & 1 & - & - & 20.6 & 9.1 & $\mathrm{CP}$ & SASC & & 2000 & \\
\hline 9 & Costa Rica & $\begin{array}{l}\text { Santuario -Caño } \\
\text { Alegre }\end{array}$ & Antioquia & 1 & - & - & 30.1 & 9 & $\mathrm{CP}$ & SASC & 1982 & 2000 & \\
\hline 1 & Cubugón & $\begin{array}{l}\text { La L ejia - } \\
\text { Saravena }\end{array}$ & $\begin{array}{l}\text { Norte de } \\
\text { Santander }\end{array}$ & 1 & - & - & 79.3 & 3.6 & A & SASC & & 2000 & \\
\hline 11 & La Honda & $\begin{array}{c}\text { Santander } \\
\text { Quilich. - Florida }\end{array}$ & Valle & 1 & - & - & 13.0 & 11.8 & $\mathrm{CR}$ & SASC & & 2000 & \\
\hline 12 & La guinea & $\begin{array}{l}\text { Santuario - Caño } \\
\text { Alegre }\end{array}$ & Antioquia & 1 & - & - & 18.8 & 9 & $\mathrm{CR}$ & SASC & & 2000 & \\
\hline 13 & Norean & $\begin{array}{c}\text { San Alberto - La } \\
\text { Mata }\end{array}$ & Ocaña & 3 & 8.05 & 20.2 & 36.3 & 12.4 & $C R$ & SASC & & 2000 & \\
\hline 14 & El Mestizo & $\begin{array}{l}\text { Sardinata - } \\
\text { Astilleros }\end{array}$ & $\begin{array}{l}\text { Norte de } \\
\text { Santander }\end{array}$ & 1 & - & - & 45.7 & 8.6 & $A C$ & SASC & & 2000 & \\
\hline 15 & Pontón & $\begin{array}{l}\text { Garzón - Río } \\
\text { Loro }\end{array}$ & Huila & 1 & - & - & 9.0 & 9.9 & $\mathrm{CR}$ & SASC & & 2000 & \\
\hline 16 & Penjamo & $\begin{array}{l}\text { Santuario - Caño } \\
\text { Alegre }\end{array}$ & Antioquia & 1 & - & - & 30.6 & 11 & $\mathrm{CR}$ & SASC & & 2000 & 2001 \\
\hline 17 & Valle -sol & $\begin{array}{l}\text { Santuario - Caño } \\
\text { Alegre }\end{array}$ & Antioquia & 1 & - & - & 31 & 9 & $\mathrm{CR}$ & SASC & & 2000 & \\
\hline 18 & El Mango & $\begin{array}{l}\text { Santuario -Caño } \\
\text { Alegre }\end{array}$ & Antioquia & 2 & 9.0 & 16.4 & 25.4 & 8.7 & CR & SASC & & 2001 & \\
\hline 19 & $\begin{array}{c}\text { Río } \\
\text { Samaná }\end{array}$ & $\begin{array}{c}\text { Santuario - Cruce } \\
\text { Ruta } 45\end{array}$ & Antioquia & 1 & - & - & 141.0 & 11.5 & $A C$ & SASV & & 2001 & \\
\hline 20 & $\begin{array}{c}\text { Paso a } \\
\text { Desnivel }\end{array}$ & Pereira-Manizales & Risaralda & 3 & 4.5 & 32.2 & 41.2 & 20 & $\mathrm{CR}$ & SASC & & 2001 & \\
\hline 21 & $\begin{array}{c}\mathrm{El} \\
\text { Caminito }\end{array}$ & $\begin{array}{l}\text { Santuario -Caño } \\
\text { Alegre }\end{array}$ & Antioquia & 1 & - & - & 20.6 & 9.1 & $\mathrm{CP}$ & SASC & & $\underset{\text { (1) }}{2001}$ & \\
\hline 22 & Cocor ná & $\begin{array}{c}\text { Santuario -Caño } \\
\text { Ruta } 45\end{array}$ & Antioquia & 3 & 21 & 31 & 73.5 & 8.4 & $\mathrm{CP}$ & VGSC & & 2001 & \\
\hline 23 & $\begin{array}{l}\text { Puente } \\
\text { Blanco }\end{array}$ & $\begin{array}{c}\text { Chigorodó - } \\
\text { Dabeiba }\end{array}$ & Antioquia & 1 & - & & 39.5 & 9.9 & $\mathrm{CP}$ & SASC & 1997 & 2002 & \\
\hline 24 & Arepas & $\begin{array}{c}\text { La Ye - Puente } \\
\text { Blanco }\end{array}$ & Boyacá & 1 & - & - & 21.3 & 9.4 & $\mathrm{CR}$ & SASC & & 2002 & \\
\hline 25 & Munar & $\begin{array}{c}\text { Bogotá - } \\
\text { Villavicencio }\end{array}$ & Cundinamarca & 1 & - & - & 30.1 & 11 & $\mathrm{CP}$ & SASC & & 2002 & \\
\hline 26 & Sardinata & $\begin{array}{c}\text { Vía al Sur del } \\
\text { Huila }\end{array}$ & Huila & 2 & 12.8 & 12.9 & 25.7 & 7.9 & $\mathrm{CP}$ & SASC & & 2002 & \\
\hline
\end{tabular}




\begin{tabular}{|c|c|c|c|c|c|c|c|c|c|c|c|c|c|}
\hline \multirow{2}{*}{ No } & \multirow{2}{*}{$\begin{array}{l}\text { Nombre } \\
\text { puente }\end{array}$} & \multirow{2}{*}{ Carretera } & \multirow{2}{*}{ Departam. } & \multicolumn{4}{|c|}{ Luces, m } & \multirow{2}{*}{$\begin{array}{c}\text { Ancho } \\
\text { tablero, } \\
\text { m }\end{array}$} & \multirow{2}{*}{$\begin{array}{l}\text { Mat. } \\
\text { constr. }\end{array}$} & \multirow{2}{*}{$\begin{array}{l}\text { Estruct. } \\
\text { long it. }\end{array}$} & \multicolumn{3}{|c|}{ Año } \\
\hline & & & & $\mathrm{N}$ & Min. & Máx. & Total & & & & Const. & Colap. & Reh. \\
\hline 27 & Guandinosa & $\begin{array}{c}\text { Garzón - Río } \\
\text { Loro } \\
\end{array}$ & Huila & 1 & - & - & 18.2 & 9.1 & $\mathrm{CR}$ & SASC & & 2002 & \\
\hline 28 & $\begin{array}{l}\text { Zanja } \\
\text { Honda }\end{array}$ & $\begin{array}{c}\text { La Paz - San Juan } \\
\text { del Cesar }\end{array}$ & Guajira & 1 & - & - & 12.0 & 11.2 & $\mathrm{CR}$ & SASC & 1958 & 2002 & 2002 \\
\hline 29 & $\begin{array}{l}\text { Molino } \\
\text { Viejo }\end{array}$ & Hatillo - Cisneros & Antioquia & 1 & - & - & 10.5 & 9.7 & $\mathrm{CR}$ & SASC & & 2002 & \\
\hline 30 & $\begin{array}{c}\text { Las } \\
\text { Animas }\end{array}$ & $\begin{array}{l}\text { San Roque - } \\
\text { Bosconia }\end{array}$ & Cesar & 1 & - & - & 15.0 & 8.1 & $\mathrm{CP}$ & SASC & & 2002 & \\
\hline 31 & Simití & $\begin{array}{c}\text { San Roque - } \\
\text { Bosconia }\end{array}$ & Cesar & 1 & - & - & 20.9 & 8.9 & $\mathrm{CR}$ & SASC & & 2002 & \\
\hline 32 & Alcaraván & $\begin{array}{c}\text { Uribe - Ye de } \\
\text { Granada }\end{array}$ & Meta & 2 & 38.4 & 115.5 & 153.9 & 11.1 & $A C$ & SASC & & $\begin{array}{c}2002 \\
\text { (1) }\end{array}$ & 2002 \\
\hline 33 & $\begin{array}{c}\text { Quebrada } \\
\text { Guasimillas }\end{array}$ & $\begin{array}{l}\text { Entre municipios } \\
\text { Hobo y Gigante }\end{array}$ & Huila & 2 & 12.6 & 12.3 & 24.5 & 11.5 & $\mathrm{CR}$ & SASC & & 2002 & \\
\hline 34 & Villapinzón & Bogotá - La Caro & Boyacá & 1 & - & - & 16.6 & 11.3 & $\mathrm{CP}$ & SASC & & 2002 & \\
\hline 35 & Guasi millas & $\begin{array}{l}\text { Garzón - Río } \\
\text { Loro }\end{array}$ & Huila & 2 & 12.2 & 12.3 & 24.5 & 11.5 & $\mathrm{CP}$ & SASC & & 2002 & \\
\hline 36 & El avispero & Suaza - Florencia & Huila & 2 & 25.2 & 92.2 & 117.3 & 7.9 & $A C$ & PSC & & 2002 & \\
\hline 37 & Morichito & $\begin{array}{l}\text { Fuente Oro - San } \\
\text { Jose Guaviare }\end{array}$ & Meta & 1 & - & - & 33.5 & 9.0 & $A C$ & SASV & & 2002 & 2004 \\
\hline 38 & Caño Largo & $\begin{array}{l}\text { La Mata - San } \\
\text { Roque }\end{array}$ & Cesar & 1 & - & - & 17.2 & 11.2 & $\mathrm{CR}$ & SASV & & 2002 & \\
\hline 39 & Pericongo & Pitalito - Garzón & Huila & 1 & - & - & 12.7 & 14.7 & $\mathrm{CR}$ & SA & & 2002 & \\
\hline 40 & $\begin{array}{l}\text { Zanjón del } \\
\text { Diablo }\end{array}$ & $\begin{array}{l}\text { Pericongo - } \\
\text { Altamira }\end{array}$ & Huila & 1 & - & - & 12.7 & 14.8 & $\mathrm{CP}$ & SASC & & 2002 & \\
\hline 41 & Sucre & $\begin{array}{l}\text { Altamira - } \\
\text { Gabinete }\end{array}$ & Caquetá & 1 & 10.9 & 10.9 & 10.9 & 7 & CR & SASC & & 2002 & \\
\hline 42 & Morichito & $\begin{array}{l}\text { Cruce P. Rico - } \\
\text { Yé Granada }\end{array}$ & Meta & 1 & 33.5 & 33.5 & 33.5 & 9.02 & $A C$ & SA & & 2002 & \\
\hline 43 & $\begin{array}{l}\text { Zanja } \\
\text { Honda }\end{array}$ & $\begin{array}{c}\text { Orrapihuasi - El } \\
\text { Vergel }\end{array}$ & Caquetá & 1 & 3 & 3 & 3 & 8.3 & $\mathrm{CR}$ & SASC & & 2002 & \\
\hline 44 & $\begin{array}{c}\text { Río } \\
\text { Mazamorra }\end{array}$ & $\begin{array}{l}\text { Popayán - La } \\
\text { Portada }\end{array}$ & Cauca & 1 & 40 & 40 & 40 & 4.6 & A & SASC & & 2002 & \\
\hline 45 & $\begin{array}{c}\text { Los } \\
\text { Esclavos } \\
\end{array}$ & $\begin{array}{l}\text { Santander Quil. - } \\
\text { Florida } \\
\end{array}$ & Cauca & 8 & 3 & 11 & 76.5 & 5.6 & M & SASC & & 2002 & \\
\hline 46 & Guaitara & $\begin{array}{c}\text { Rumichaca - } \\
\text { Pasto }\end{array}$ & Nariño & 3 & 17.3 & 30.9 & 67.1 & 8.1 & $\mathrm{CR}$ & SASV & & 2002 & \\
\hline 47 & Quebradón & $\begin{array}{l}\text { Vía comunica con } \\
\text { el Doncello }\end{array}$ & Caquetá & 1 & 17 & 17 & 17 & 8.6 & $\mathrm{CP}$ & SASC & & 2002 & \\
\hline 48 & Montecristo & $\begin{array}{c}\text { Florencia - Puerto } \\
\text { Rico }\end{array}$ & Caquetá & 3 & 30.1 & 30.2 & 90.4 & 8.05 & $\mathrm{CR}$ & SASC & & 2002 & \\
\hline 49 & Montañita & $\begin{array}{l}\text { Ví a del Paujil - } \\
\text { Doncello }\end{array}$ & Caquetá & 2 & 9.6 & 21.2 & 30.4 & 7.9 & $\mathrm{CR}$ & SASC & & 2002 & \\
\hline 50 & $\begin{array}{c}\text { Santa } \\
\text { Helena }\end{array}$ & Neiva - Balsillas & Huila & 1 & 14.9 & 14.9 & 14.9 & 6.5 & $\mathrm{CR}$ & SASC & & 2002 & \\
\hline 51 & Cayumba & Puerto Wilches & Santander & 1 & 31.4 & 31.4 & 31.4 & 11.6 & $\mathrm{CP}$ & SASC & & 2002 & \\
\hline 52 & Mazamorras & $\begin{array}{c}\text { Puracé - } \\
\text { Quebrada blanca }\end{array}$ & Cauca & 1 & 40 & 40 & 40 & 4.6 & A & VCSC & & 2003 & 2008 \\
\hline
\end{tabular}




\begin{tabular}{|c|c|c|c|c|c|c|c|c|c|c|c|c|c|}
\hline \multirow{2}{*}{ No } & \multirow{2}{*}{$\begin{array}{l}\text { Nombre } \\
\text { puente }\end{array}$} & \multirow{2}{*}{ Carretera } & \multirow{2}{*}{ Departam. } & \multicolumn{4}{|c|}{ Luces, m } & \multirow{2}{*}{$\begin{array}{c}\text { Ancho } \\
\text { tablero, } \\
\text { m }\end{array}$} & \multirow{2}{*}{$\begin{array}{l}\text { Mat. } \\
\text { constr. }\end{array}$} & \multirow{2}{*}{$\begin{array}{l}\text { Estruct. } \\
\text { long it. }\end{array}$} & \multicolumn{3}{|c|}{ Año } \\
\hline & & & & $\mathrm{N}$ & Min. & Máx. & Total & & & & Const. & Colap. & Reh. \\
\hline 53 & $\begin{array}{c}\text { Río } \\
\text { Guamez }\end{array}$ & $\begin{array}{c}\text { San Miguel - } \\
\text { Santa Ana }\end{array}$ & Putumayo & 3 & 17.3 & 54.2 & 101.5 & 4 & $A C$ & SASC & & 2003 & \\
\hline 54 & Amarón & $\begin{array}{l}\text { San Miguel - } \\
\text { Santa Ana }\end{array}$ & Putumayo & 1 & 11.4 & 11.4 & 11.4 & 7.9 & $A C$ & SASV & & 2004 & \\
\hline 55 & Guayas & $\begin{array}{l}\text { Puerto Rico - San } \\
\text { Vicente }\end{array}$ & Caquetá & 4 & 28.9 & 60.5 & 159.7 & 8 & $A C$ & VCSC & 1982 & $\underset{\text { (1) }}{2004}$ & 2005 \\
\hline 56 & $\begin{array}{c}\text { Río } \\
\text { Villalobos }\end{array}$ & Mocoa - Pitalito & Putumayo & 2 & 16 & 60 & 76 & 9 & CR & SASC & 1990 & 2005 & \\
\hline 57 & El Tarra & Ocaña - Sardinata & Ocaña & 1 & 28.2 & 28.2 & 28.2 & 11 & $\mathrm{CR}$ & SASV & & 2006 & \\
\hline 58 & Pororio & $\begin{array}{c}\text { San V. Guaviare - } \\
\text { Puerto Rico }\end{array}$ & Guaviare & 3 & 8.6 & 33.8 & 51.1 & 9.6 & $\mathrm{CR}$ & SASV & & 2012 & \\
\hline 59 & $\begin{array}{c}\text { Río } \\
\text { Piendamó }\end{array}$ & Popayán - Cali & Cauca & 1 & 25.6 & 25.6 & 25.6 & 12.2 & CR & SASC & & 2012 & \\
\hline 60 & Riecito & $\begin{array}{l}\text { Puerto Rico - San } \\
\text { V. del Caguá n }\end{array}$ & Caquetá & 1 & 61.6 & 61.6 & 62.7 & 7.9 & $A C$ & SASC & 2004 & 2012 & 2012 \\
\hline 61 & Guayas & $\begin{array}{l}\text { Puerto Rico - San } \\
\text { V. del Caguá n }\end{array}$ & Caquetá & 4 & 28.9 & 60.5 & 159.7 & 8 & $A C$ & VCSC & 1982 & $\underset{\text { (1) }}{2012}$ & \\
\hline 62 & Río Ovejas & Popayán - Cali & Cauca & 1 & 25.7 & 25.7 & 25.7 & 10.9 & CR & PSC & & 2012 & \\
\hline 63 & El guajiro & $\begin{array}{l}\text { Barrancas - } \\
\text { Fonsecas }\end{array}$ & Guajira & 1 & 32 & 32 & 32 & 9.3 & CR & SASC & 1958 & 2013 & \\
\hline 64 & $\begin{array}{c}\text { Río } \\
\text { Mandiva }\end{array}$ & Popayán - Cali & Cauca & 1 & 19 & 19 & 19 & 12.3 & $\mathrm{CR}$ & SASC & & 2013 & \\
\hline 65 & $\begin{array}{l}\text { Caño } \\
\text { Ovejas }\end{array}$ & Mapiripan - Meta & Villavicencio & 1 & 60 & 60 & 60 & 8.5 & $\begin{array}{c}\text { C.ASF. } \\
\text { BM }\end{array}$ & SASC & 2012 & 2014 & \\
\hline 66 & Rí o Negro & $\begin{array}{l}\text { La Lejía - } \\
\text { Saravena }\end{array}$ & $\begin{array}{l}\text { Norte de } \\
\text { Santander }\end{array}$ & 3 & 11.4 & 20 & 42.9 & 7.7 & $\mathrm{CR}$ & SASC & & 2014 & \\
\hline 67 & Cocorná & $\begin{array}{c}\text { Santuario - Caño } \\
\text { Ruta } 45\end{array}$ & Antioquia & 3 & 21 & 31 & 73.4 & 8.4 & $\mathrm{CP}$ & VGSC & & 2001 & \\
\hline
\end{tabular}

(1) Puente colapsado dos veces.

tenía como material de construcción el concreto entre 1993 y 2014, 67 son conocidos por el reforzado, CR. Por ejemplo, como se muestra SIPUCOL y 31 de ellos (46\%) fueron construidos en la Figura 4, de los 115 puentes registrados con CR.

Tabla 3. Puentes atentados y colapsados desde 1994 hasta 2014 no registrados en SIPUCOL.

\begin{tabular}{|c|c|c|c|c|}
\hline No & Nombre del puente & Carretera & Departamento & $\begin{array}{c}\text { Año de } \\
\text { colapso }\end{array}$ \\
\hline 1 & Guanabano & Riohacha - Valledupar & Guajira & $1996(1)$ \\
\hline 2 & La Hamaca & Ve reda Buenos Aires & Norte de Santander & 2000 \\
\hline 3 & $\begin{array}{c}\text { Sobre Río Nuevo } \\
\text { Presidente }\end{array}$ & Tibú - Cauca & Norte de Santander & 2000 \\
\hline 4 & Caño Raya & Tibú - Cauca & Norte de Santander & 2002 \\
\hline
\end{tabular}




\begin{tabular}{|c|c|c|c|c|}
\hline No & Nombre del puente & Carretera & Departamento & $\begin{array}{l}\text { Año de } \\
\text { colapso }\end{array}$ \\
\hline 5 & $\begin{array}{l}\text { Sobre Quebrada } \\
\text { Trinidad }\end{array}$ & Pios - Cocorná & Antioquia & 2000 \\
\hline 6 & $\begin{array}{c}\text { Sobre Quebrada } \\
\text { Cascajal }\end{array}$ & Municipio San Luis & Antioquia & 2000 \\
\hline 7 & La unión & $\begin{array}{c}\text { Vía cor regimiento Santa } \\
\text { Cecilia }\end{array}$ & Risaralda & 2000 \\
\hline 8 & (No Registra) & Barbosa - Santo Domingo & Antioquia & 2001 \\
\hline 9 & Papacito Aguasmal & Vía Arauquita & Arauca & 2002 \\
\hline 10 & (No Registra) & Vereda Marrero - Tame & Arauca & 2002 \\
\hline 11 & (No Registra) & Quebrada las Cardo nas & Antioquia & 2002 \\
\hline 12 & (No Registra) & $\begin{array}{c}\text { Vía Inspección Policía el } \\
\text { Hatillo } \\
\end{array}$ & Antioquia & 2000 \\
\hline 13 & (No Registra) & Vía Segovia - Saragoza & Antioquia & 2000 \\
\hline 14 & La Flor & Danticas - San Rafael & Antioquia & 2002 \\
\hline 15 & (No Registra) & $\begin{array}{c}30 \text { km (embalse de la } \\
\text { hidroelé ctrica San C arlos) }\end{array}$ & Antioquia & 2002 \\
\hline 16 & (No Registra) & Sobre la Quebrada Bizcocho & Antioquia & 2002 \\
\hline 17 & (No Registra) & Vía corregimiento Tucurinca & Magdalena & 2000 \\
\hline 18 & Quitiva & $\begin{array}{c}\text { San Juan de Arama - Vista } \\
\text { Hermosa }\end{array}$ & M eta & 2002 \\
\hline 19 & (No Registra) & Ariari -Villavicencio & Meta & 2002 \\
\hline 20 & Porporio & $\begin{array}{c}\text { Puerto Rico - Puerto } \\
\text { Concordia }\end{array}$ & Caquetá & 2002 \\
\hline 21 & El Portón & Vereda Arenosa del Doncello & C aquetá & 2002 \\
\hline 22 & Quebradona & Puerto Rico - Florencia & Caquetá & 2002 \\
\hline 23 & Currillo & Via Currillito - El Dorado & Caquetá & 2002 \\
\hline 24 & Tablitas & $\begin{array}{l}\text { La Jagua De Ibirico - San } \\
\text { Roque }\end{array}$ & Cesar & 2002 \\
\hline 25 & (No Registra) & $\begin{array}{c}\text { Vía Inspección Policía } \\
\text { Mazamorras }\end{array}$ & Cauca & 2000 \\
\hline 26 & (No Registra) & $\begin{array}{c}\text { Inspecci ón De Policía } \\
\text { Villalobos } \\
\end{array}$ & Cauca & 2002 \\
\hline 27 & La virgen & Corregimiento Victoria & Nariño & 2002 \\
\hline 28 & Puente Viejo & Corregimiento Las Lajas & Nariño & 2002 \\
\hline 29 & Río Aguacatal & $\begin{array}{l}\text { Inspecci ón de Polic ía } \\
\text { Gallardo }\end{array}$ & Huila & 2002 \\
\hline 30 & Río Caunce & Pensilvania - Manzanares & Caldas & 2002 \\
\hline 31 & La Peña & Jurisdicción de La Palma & Cundinamarca & 2002 \\
\hline 32 & (No Registra) & Casco Urbano San Benito & Sucre & 2002 \\
\hline 33 & (No Registra) & Quebrada El Choco & Choco & 2003 \\
\hline 34 & Campo Hermoso & $\begin{array}{l}\text { Ver eda Las Juntas - Vía } \\
\text { Florencia }\end{array}$ & Huila & 2003 \\
\hline
\end{tabular}




\begin{tabular}{|c|c|c|c|c|}
\hline No & Nombre del puente & Carretera & Departamento & $\begin{array}{c}\text { Año de } \\
\text { colapso }\end{array}$ \\
\hline 35 & La Quiebra & V ía San Francisco - Medellín & Antioquia & 2004 \\
\hline 36 & (No Registra) & Norte de Mocoa & C aquetá & 2004 \\
\hline 37 & Rio Tenerife & $\begin{array}{c}\text { Inspección de Policía El } \\
\text { Silencio }\end{array}$ & Caldas & 2004 \\
\hline 38 & Naranjito & Vía Mocoa - Puerto Asís & Putumayo & 2005 \\
\hline 39 & Rio Manso & $\begin{array}{c}\text { Corregimientos Berlín y San } \\
\text { Diego }\end{array}$ & Caldas & 2006 \\
\hline 40 & Rio Perrito & $\begin{array}{c}\text { Comunica el Departamento } \\
\text { del Tolima }\end{array}$ & Tolima & 2006 \\
\hline 41 & (No Registra) & Corregimiento Teteyé & Putumayo & 2006 \\
\hline 42 & El Tabalazo & Cúcuta - Tibú & Norte de Santander & 2009 \\
\hline 43 & La Unión & Guadalupe - Anorí & Antioquia & 2011 \\
\hline 44 & Cañón Cussay & Vereda Puerto Jordán & Cauca & 2012 \\
\hline 45 & Campo Dos & Cúcuta - Tibú & Norte de Santander & 2012 \\
\hline 46 & La Hamaca & Vereda Buenos Aires & Norte de Santander & 2012 \\
\hline 47 & Puente López & Sobre Río Neiva & Neiva & 2013 \\
\hline 48 & Río Neiva & Campo Alegre - Hobo & Neiva & 2013 \\
\hline
\end{tabular}

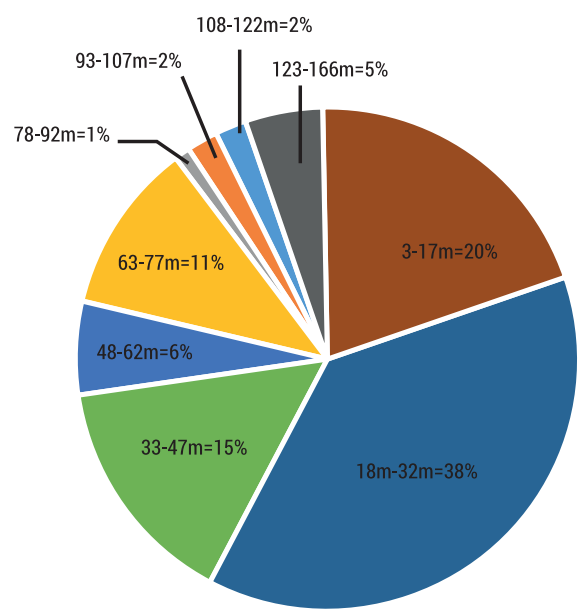

Figura 3. Longitudes de los puentes colapsados entre 1993-2014 reconocidos por SIPUCOL.

Para el grupo Marte de la Escuela de y costosas, pues se deben utilizar cantidades Ingenieros Militares, la rehabilitación de los considerables de explosivos para terminar su puentes construidos con concreto reforzado colapso. Además, ellargotiempoproporcionado se considera como una de las más tardadas a esta actividad genera exposición de la 
fuerza pública a nuevos posibles ataques y a sabotajes de la guerrilla para no permitir su rehabilitación.

La estructuración longitudinal del puente es parte importante de la estrategia de colocación del explosivo pues, entre más largo sea el puente, su colapso es más desfavorable y perjudicial para la población. Por ejemplo, la longitud e importancia del puente "Uribe Ye" de Granada, el cual tenía una longitud de 153.9 m, generó que su rehabilitación después del colapso fuera inmediata. Por otro lado, como se muestra en la Figura 5, el tipo de estructuración de los puentes colapsados que ha sido más recurrente ha sido el de puentes simplemente apoyados con sección constante, SASC. 47 Puentes conocidos y registrados tenían este tipo de estructuración $(71 \%)$. El puente simplemente apoyado es más propenso a un ataque guerrillero, pues al dinamitar un pequeño tramo del puente, todo el tablero del mismo puede colapsar.

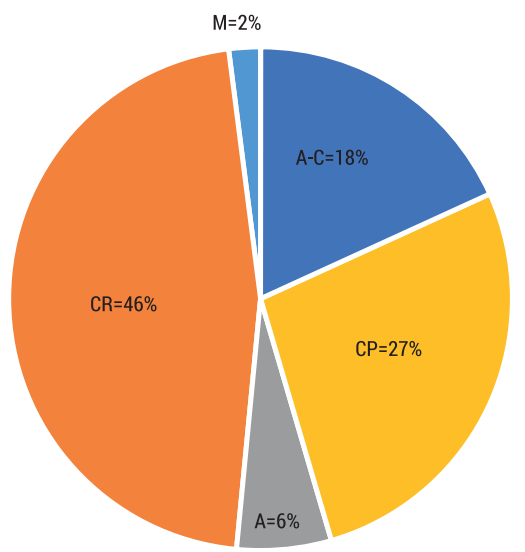

Figura 4. Material de la superestructura de los puentes colapsados entre 1993-2014.

Por razones de orden público y ubicación, tal como se indica en la Figura 6, Antioquia

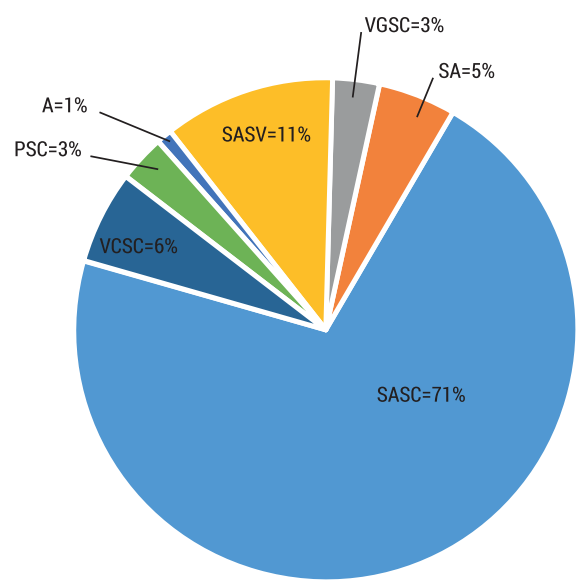

Figura 5. Estructuración longitudinal de los puentes colapsados entre 1993-2014.

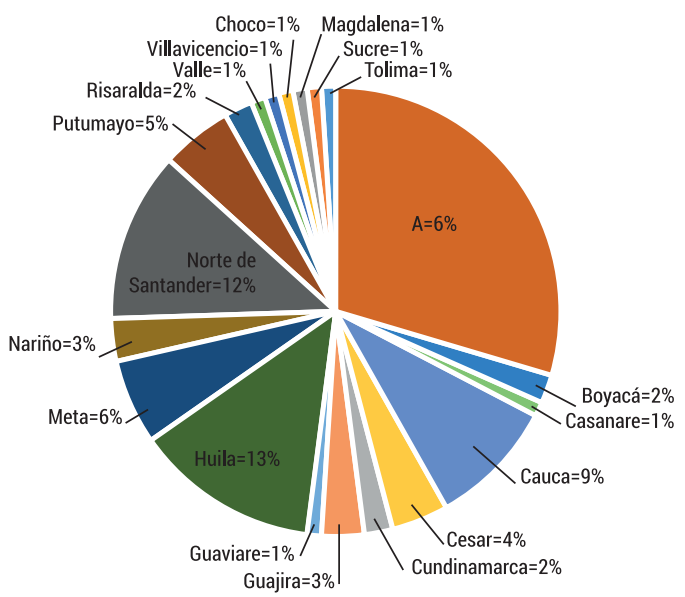

Figura 6. Ubicación de los puentes colapsados entre 1993-2014.

es el departamento más repetitivo en tener atentados terroristas contra puentes. Es ampliamente conocido que el orden público en esta región es bastante complicado y que esta zona del país tiene un compromiso económico nacional de gran magnitud, lo que lo hace un blanco de alto valor para sabotaje y ruptura de la estabilidad de la nación. 


\section{CARGAS EXPLOSIVAS UTILIZADAS EN EL COLAPSO DE PUENTES}

La instalación del explosivo es primordial para producir el efecto colateral por la onda explosiva. El tipo de explosivo y su cantidad, las características técnicas y de colocación sobre la subestructura o superestructura del puente, el material y medidas del objeto a colapsar son puntos determinantes que son previamente analizados por los grupos terroristas para la adecuación del explosivo en la estructura, ya que el acto delictivo terrorista tiene presente el daño y la ubicación de mayor impacto contra la estructura.

Los explosivos más utilizados por los grupos terroristas colombianos para derribar puentes son el anfo y la pentolita. El anfo es la combinación de nitrato de amonio, producido comúnmente como fertilizante con Fuel-Oil o cualquier derivado del petróleo. La pentolita es la combinación de TNT y pentrita. Estos materiales explosivos se comparan de acuerdo con el factor de efectividad o potencia explosiva, Fer. Este factor indica la efectividad de la energía de los gases de explosión de la carga; por ejemplo, en el caso del TNT, este factor es 1, para el anfo, el Fer posee una efectividad menor de 0,80, y para la pentolita, el Fer es de 0,30, lo cual significa que estos son los materiales con mayor efectividad para colapsar un puente.
La selección de la vía de destrucción de un puente comprende una atención estratégica dirigida al desgaste de propias tropas y de los servidores públicos. Para los grupos terroristas, el uso «adecuado» o «inadecuado» de los escombros se representa en la Figura 7.

De acuerdo con la Figura. 7, para los grupos al margen de la ley el modo adecuado de utilización de escombros consiste en dejar que gran parte del puente quede en forma de obstáculo y represente un desgaste tanto económico como de personal para iniciar el proceso de restablecimiento de operación o de rehabilitación. Para que este modo de utilización de escombros sea efectivo para los grupos terroristas, el mecanismo de colapso que ellos definen como el «más indicado» para delinquir es el mecanismo de colapso de viga, que se representa en la Figura 8.

El mecanismo por colapso de viga es altamente efectivo para estos grupos, pues como resultado la superestructura se comporta como un obstáculo de gran magnitud y de bastante tiempo de remoción, que es la finalidad buscada por el terrorista que instala y acciona el explosivo. Para efectos del concreto reforzado, estos grupos buscan que el refuerzo principal sea deformado y que el refuerzo secundario sea cortado. La efectividad de la explosión produce grietas en

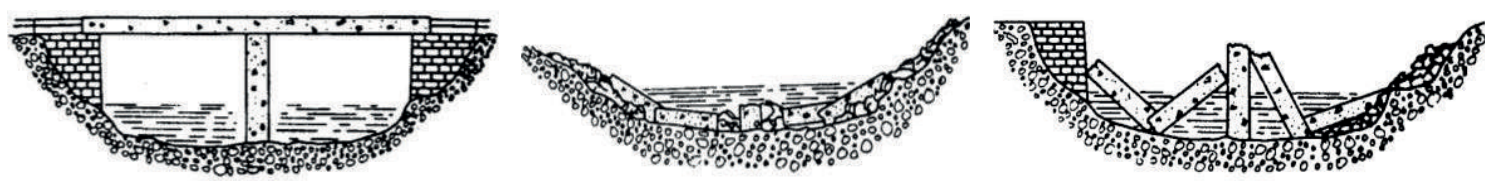

Figura 7. Utilización de escombros de un puente para actos terroristas: (a) antes del ataque, (b) uso «inapropiado» de escombros, (c) uso «apropiado» de escombros.

Fuente: ICSH, 1998 [3] 

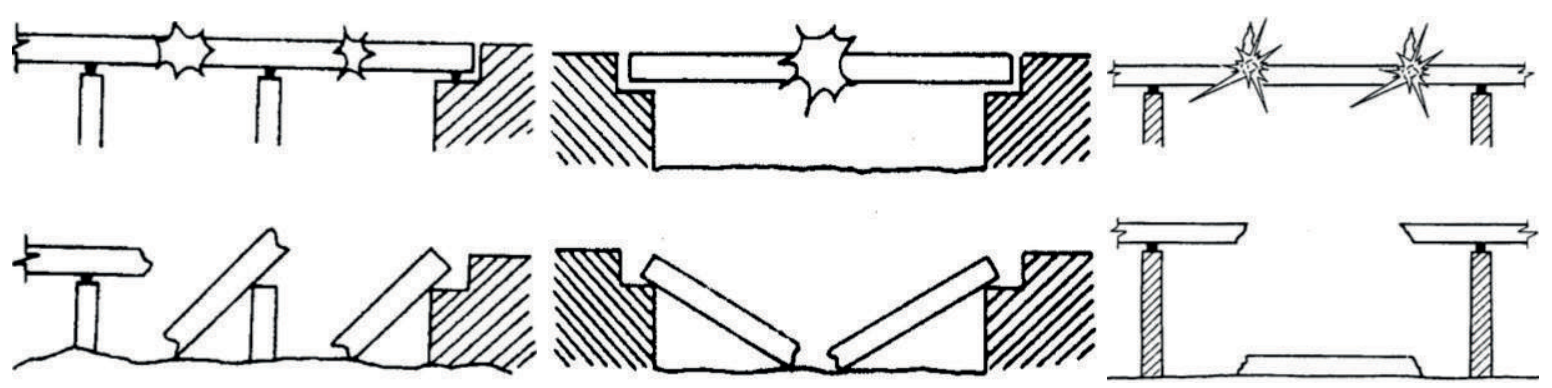

Figura 8. Tipos de mecanismos de colapso: (a) colapso oscilante, (b) colapso de viga, (c) colapso de miembro sin apoyo. Fuente: ICSH, 1998 [3].

el concreto, las cuales ayudan a que el refuerzo se debilite, dando entonces paso al colapso de la estructura.

\section{IMPACTOS PRODUCIDOS POR COLAPSO DE PUENTES POR ATENTADOS TERRORISTAS}

Según la Organización Inspiraction [13], el colapso de un puente por causas terroristas ha generado los siguientes impactos a nivel social, político y económico en Colombia:

- Desplazamiento forzado de campesinos de sus tierras y hogares, provocando una de las mayores crisis humanitaria y social que Colombia ha podido enfrentar. Esto afecta seriamente la psicología de la persona desplazada, pues se tardan muchos años en recuperar la normalidad.

- Desintegración familiar y desempleo, pues muchas familias han tenido que enviar a sus hijos a las grandes ciudades en busca de oportunidades de empleo. Las personas que no corren con esta suerte reportan que una de las únicas salidas que tienen es pertenecer a alguna de las filas de los grupos armados al margen de la ley.
- Crisis al desarrollo económico, puesto quelas consecuencias sociales del conflicto armado colombiano son siempre desfavorables y no ayudan a atraer capital al país, generando desconfianza a los empresarios locales e internacionales en su interés de inversión.

- Pérdidas millonarias dirigidas a actividades de labor social. Las obras por desastres naturales y obras humanitarias usualmente se cancelan para poder habilitar un puente colapsado por atentados terroristas. Por ejemplo, como se muestra en la tabla 4, dentro de las causas de pérdidas por riesgos extraordinarios, los daños terroristas representan al menos un $8,7 \%$, lo que los ubica en segundo lugar después de las inundaciones.

- Decrecimiento constante del sector primario de la economía nacional por pérdidas de insumos imposibles de transportar en vías alternas a la del puente derrumbado.

- Intervención riesgosa de instituciones como INVIAS, quienes no sólo deben asesorar y controlar la reconstrucción del puente, sino que también deben enfrentar la posición de peligro referida a la seguridad personal, pues el sabotaje es continuo durante la rehabilitación del puente. 
Tabla 4. Porcentaje de pérdidas por riesgos extraordinarios

\begin{tabular}{|c|r|}
\hline Causas & $\%$ de pérdidas \\
\hline Inundaciones & $85.8 \%$ \\
Terremotos & $1.4 \%$ \\
Volcanes & $0.0 \%$ \\
Vientos & $0.6 \%$ \\
Meteoritos & $0.0 \%$ \\
Terrorismo & $8.7 \%$ \\
Rebelión & $0.1 \%$ \\
Conmoción Civil & $3.4 \%$ \\
Actos fuerzas Armadas & $0.1 \%$ \\
\hline TOTAL & $100.0 \%$ \\
\hline
\end{tabular}

Fuente: Arango, 2003 [12]

- Los altos mandos del Estado consiguen una popularidad desquebrajada por no satisfacer las necesidades de la población en el momento en que son solicitadas por la comunidad. La educación, alimentación, seguridad y transporte llegan a ser los servicios más afectados por los atentados terroristas.

- Necesidad de desplazamiento de una zona a otra, lo que genera una sobrepoblación no concebida.

- Consolidación terrorista en el tema de seguridad nacional, permitiendo la debilidad de las fuerzas militares y daños a la estructura de defensa del territorio nacional.

- Instalación de retenes guerrilleros sobre las vías alternas, generando robos, saqueos, secuestros y asesinatos seleccionados.

- Disminución de la infraestructura vial del país, ya que los ingenieros y constructoras evitan algún tipo de trabajo sobre zonas de alto riesgo delictivo.

\section{CASO DE ESTUDIO: PUENTE RIECITO EN CAQUETÁ}

El puente Riecito, localizado en el departamento del Caquetá, fue colapsado por la «Columna Teófilo Forero» de las FARC, empleando explosivos de alto poder destructivo, el día 20 de julio del año 2012 aproximadamente a las 3:30 am. El puente cumplía con la función vital de comunicar el municipio de Puerto Rico con San Vicente del Caguán. La intención de las FARC al atentar contra este puente era dejar incomunicado al municipio de San Vicente del Caguán con el oriente del país.

Este puente contaba con longitud de 62,7 m, ancho de 8,3 m, y tenía como soporte estribos sobre la cuenca del río. En la Figura 5 se muestra la sección longitudinal y transversal del puente. La vía que el puente tenía como conector era de $536 \mathrm{~km}$ y comunicaba los dos municipios. La estructura se encontraba construida a 23 kilómetros del municipio de Puerto Rico.

Como se muestra en las Figuras 10 y 11, el daño causado a esta estructura por las FARC estaba estratégicamente planeado para que las partes del puente destruidas sirvieran de obstáculo al transitar por el afluente. Además, se buscaba que la construcción de una nueva estructura fuera dificultosa para el Ejército Nacional e INVIAS, pues la superestructura del puente se desplomó de forma inclinada hasta llegar al fondo del río.

El colapso del puente generó muchos impactos intencionales a la población y al Estado, pues se afectó significativamente la tranquilidad, la 


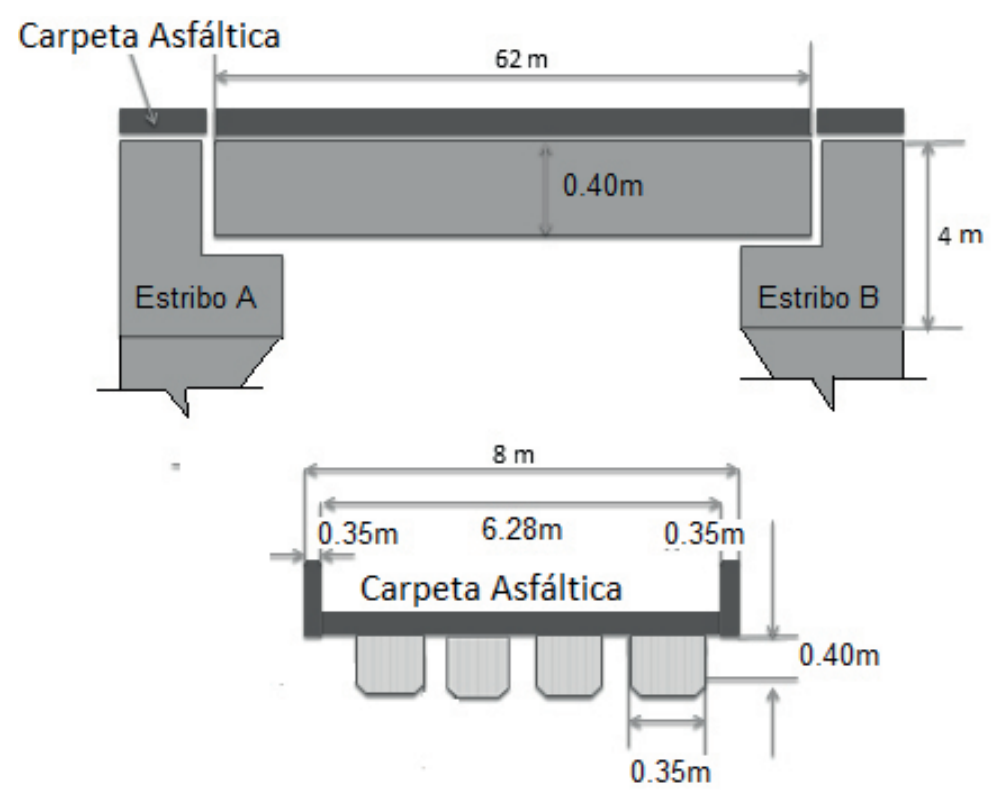

Figura 9. Dimensiones del Puente Riecito.

seguridad y el bienestar social, al punto de que las producciones de petróleo y sus derivados perdieran un monto diario aproximado de 3.5 millones de USD por concepto de regalías. Adicionalmente, no se logró transportar la leche y se desaprovecharon aproximadamente 11000 litros de ésta. Por otro lado, la crisis generada en la región desmotivó notoriamente al sector primario, pues la producción y el transporte de productos alimenticios tradicionales se detuvieron y, por tanto, los índices económicos del Caquetá cayeron significativamente.

El puente Riecito es de gran importancia para el departamento del Caquetá. Por tanto, se necesitó la intervención inmediata del Ejercito Nacional e INVIAS para solucionar la necesidad de la comunidad. Los ingenieros militares intercedieron en la construcción de un puente tipo Acrow, de configuración triple-doble reforzado, con longitud de $67,1 \mathrm{~m}$, y habilitado para un solo carril con una capacidad de soporte de 510 kN aproximadamente. El Grupo Marte de la división del departamento de Caquetá llevó a cabo la demolición con explosivos de las partes restantes del puente colapsado. El INVIAS cumplió un papel fundamental en las siguientes actividades: conformación de una variante, colocación de geotexiles en los terraplenes, reconstrucción del estribo destruido, placas de acceso, elementos de apoyo en concreto, terraplenes de acceso y estructuras de contención, entre otras. El día 20 de agosto del año 2012, un mes después de su demolición, se hizo entrega del puente militar a la comunidad del Caquetá (ver Figura 12).

\section{CONCLUSIONES}

En este artículo se han evaluado estadísticamente los datos registrados del colapso de puentes en Colombia en el periodo 


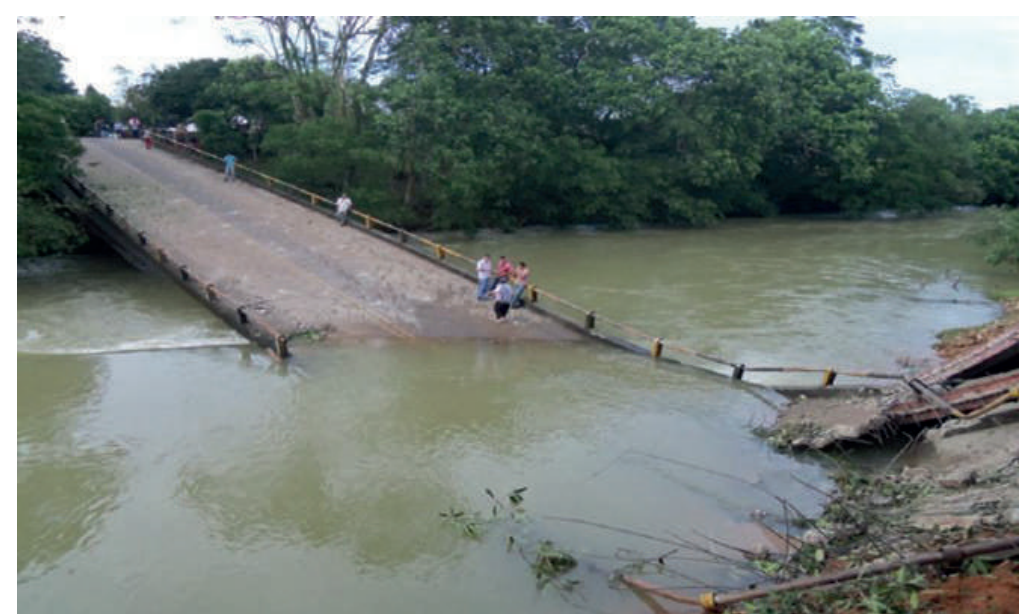

Figura 10. Panorámica lateral del puente Riecito luego del colapso.

Fuente: INVIAS, 2012 [11]

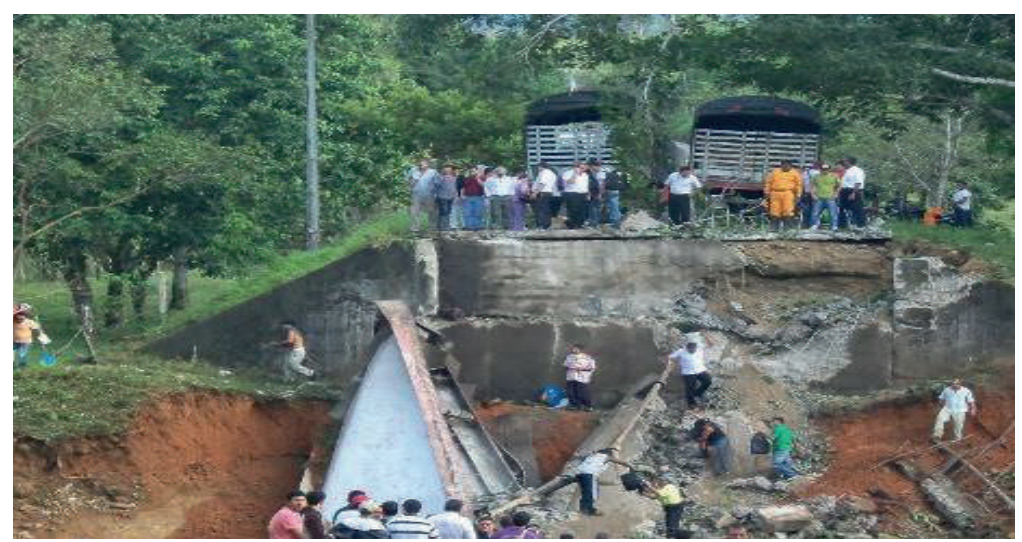

Figura 11. Panorámica del estribo sur del puente Riecito luego del colapso.

Fuente: INVIAS, 2012 [11]

de 1994 a 2014. Con los datos recolectados y las estadísticas previamente consultadas y analizadas se logró comprobar que la cantidad de puentes atentados en el año 2002 fue incentivada por la terminación de la zona de distención establecida entre el Gobierno y las FARC. Después de comparar las cifras recolectadas desde 1994 hasta 2014 fue posible comprobar que la cantidad de puentes varía dependiendo del periodo presidencial y la gestión de seguridad impartida por los mandatarios.

Los puentes colapsados por acciones terroristas tienen un proceso de análisis y una capacitación previa para su colapso exitoso. Es por ello que en el periodo del 2007 al 2014, los índices de atentados contra puentes fueron bajos con respecto a los otros años, pues la capacitación del cuerpo terrorista fue 


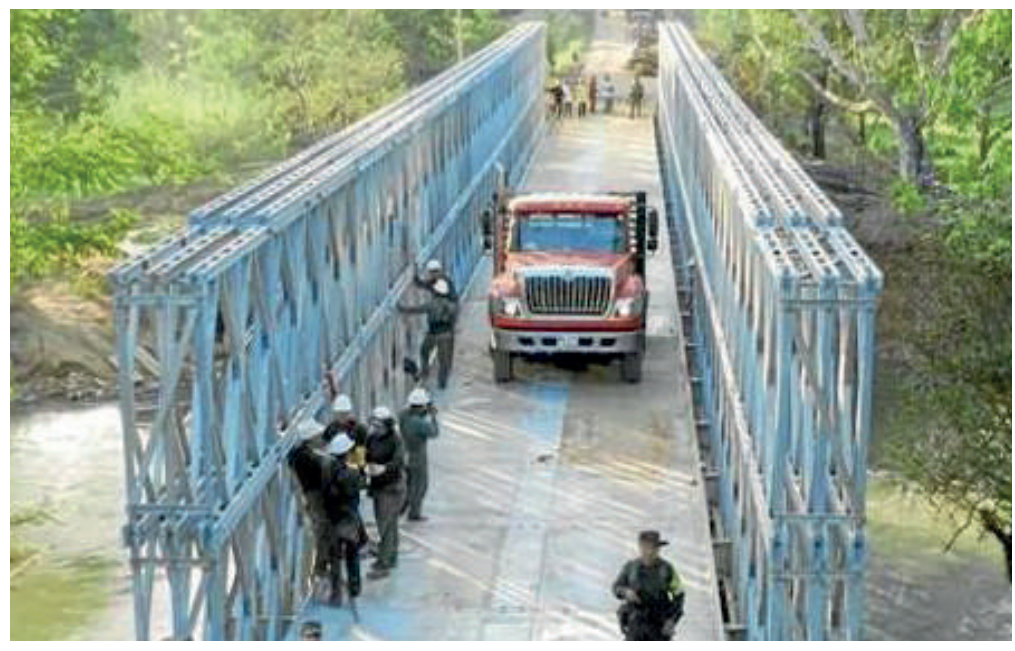

Figura 12. Puente Acrow en configuración triple-doble para remplazar el puente Riecito destruido por grupos terroristas.

Fuente: INVIAS, 2012 [11].

combatida y deteriorada por un gran número de estrategias planteadas para mejorar la seguridad nacional.

La reconstrucción de los puentes colapsados detiene los planes de mantenimiento y desarrollo vial del país, puesto que la necesidad genera una intervención rápida que desvía la atención de los recursos ya previamente asignados a una emergencia extraordinaria provocada. El daño estructural de los puentes por explosivos es básicamente imposible de combatir si no se tiene un plan de seguridad territorial que aleje al personal relacionado con grupos al margen de la ley. El poder destructivo de una carga terrorista está diseñado para crear bastante deterioro a la estructura del puente y genera trabajos de desgaste adicionales a los que comúnmente existen en un proceso de rehabilitación constructiva.

La intervención de las fuerzas militares en cuanto al suministro de los puentes móviles tipo Acrow es de gran importancia para la labor social del país, puesto que estos puentes son adquiridos para uso del ejército, y tienen una razón netamente operacional. Estos puentes son utilizados y entregados a la comunidad para su servicio, en búsqueda de una solución pronta y efectiva de los daños ocurridos por los atentados terroristas. Por ejemplo, debido a la implementación del puente Acrow en el sector donde antes se encontraba el puente Riecito, las cifras económicas ascendieron notoriamente después de haber sido víctimas de descensos y pérdidas millonarias. La variante habilitada por el INVIAS es un soporte para esta zona del país, puesto que el tránsito de vehículos pesados continuamente sobre el puente Acrow de un solo carril podría ser algo difícil y tedioso. En general, estos puentes de rehabilitación son dados a la población civil por emergencia, lo cual hace que la variante se convierta en otro curso de acción o vía a tomar por la población beneficiada en este sector. 


\section{AGRADECIMIENTOS}

Los autores agradecen a la Vicerrectoría de Investigaciones de la Universidad Militar Nueva Granada, UMNG, por el apoyo económico del proyecto IMP-ING-1574 y a la Jefatura de Ingenieros Militares de Colombia por proporcionar parte de la información para el artículo. El contenido de este artículo representa exclusivamente la opinión de los autores y no refleja la opinión de los patrocinadores y/o colaboradores.

\section{REFERENCIAS BIBLIOGRAFICAS}

[1] Leal, F. (1989). Estado y política en Colombia. 2a. edición aumentada. Siglo XXI Editores.

[2] Muñoz, E. (2003). Estudio de las causas del colapso de algunos puentes en Colombia. Facultad de Ingeniería, Pontificia Universidad Javeriana, pp.1-20.

[3] Instituto de Cooperación para la Seguridad Hemisférica, ICSH. (1998). Demolición de puentes. En: FM 5-250 Explosivos y demoliciones. pp.4.1-4.38. Washington, DC, EE.UU.: Cuartel General de la Secretaría del Ejército Estadounidense.

[4] Sánchez, I. (2004). ¿Son todos los terrorismos iguales?. Claves de Razón Práctica, 144, p.22.

[5] Domínguez, E. (2002). Atacados 76 puentes ese año. El Tiempo, Redacción Nacional.

[6] Durán, I. (2011). Conflicto armado y crecimiento económico municipal en
Colombia (Tesis de maestría inédita). Universidad Nacional de Colombia, Bogotá, Colombia.

[7] Castillo, L. (2006). Aplicación de un modelo numérico de flujos de escombros y lodo en una quebrada en el Perú (Tesis de pregrado inédita). Universidad Nacional de Ingeniería, Lima, Perú.

[8] Centro Nacional de Memoria Histórica. (2013). ¡Basta Ya! Colombia. Memorias de Guerra y Dignidad. Bogotá, Colombia: Centro Nacional de Memoria Histórica, p.434.

[9] Departamento Nacional de Planeación. (2003). Atentados a puentes primer semestre 2001 - 2003. Bogotá, Colombia: DNP.

[10] Vallecilla, R. (2004). Estática para Ingenieros Civiles. Bogotá, Colombia: Universidad Santo Tomás.

[11] Instituto Nacional de Vías. (2012). INVIAS trabaja para abrir paso provisional tras caída de puente en Caquetá. En: Oficina de Comunicaciones.

[12] Arango, C. (2005). Terrorismo y seguro de terrorismo. Consideraciones de carácter jurídico, económico y social (Tesis de pregrado inédita). Pontificia Universidad Javeriana, Bogotá, Colombia.

[13] Conflicto armado en Colombia. (s.f.) Recuperado en mayo de 2004 de https://www.inspiraction.org/justiciaeconomica/conflicto-armado-encolombia 